\title{
Applications of BMI or BSI: Differences and Revisions According to Age and Height
}

\author{
Otto W. B. Schult, ${ }^{1}$ Ludwig E. Feinendegen, ${ }^{2,3}$ Stephan Zaum, ${ }^{4}$ Walton W. Shreeve, ${ }^{3}$ \\ and Richard N. Pierson Jr. ${ }^{5}$ \\ ${ }^{1}$ Research Center Jülich, Institute of Nuclear Physics (IKP), Forschungszentrum Juelich, 52425 Jülich, Germany \\ ${ }^{2}$ Department of Nuclear Medicine, Heinrich-Heine University Düsseldorf, 40225 Düsseldorf, Germany \\ ${ }^{3}$ Medical Department, Brookhaven National Laboratory, Upton, NY 11973-5000, USA \\ ${ }^{4}$ Office for Pediatrics and Youth Medicine, 52152 Simmerath, Germany \\ ${ }^{5}$ St. Luke's/Roosevelt Hospital Center, Columbia University, New York, NY 10027-6902, USA
}

Correspondence should be addressed to Otto W. B. Schult, o.schult@fz-juelich.de

Received 26 January 2010; Revised 16 June 2010; Accepted 13 July 2010

Academic Editor: Serena Tonstad

Copyright (C) 2010 Otto W. B. Schult et al. This is an open access article distributed under the Creative Commons Attribution License, which permits unrestricted use, distribution, and reproduction in any medium, provided the original work is properly cited.

\begin{abstract}
Validation of body-mass relationships requires a careful statistical analysis of data of normal weight individuals. BMI (ratio between body mass and square of body height) and BSI values (ratio between mass and cube of body height) have been calculated for 99 persons with ages between 1 day and 76 years. These BMI or BSI values have been used for least squares fits yielding mean BMI or BSI values, their variances (providing precision), and average deviations of individual BMI/BSI values from the BMI/BSI means. The latter allows limits to over- and underweight. For adults we found mean values of BSI of 12.36 and confirmed 21.7 for the mean BMI; but the BSI was 1.4 times more precise than the BMI. For children shorter than $1.3 \mathrm{~m}$ and younger than 8 years we found the BMI average of 15.9 and over-/underweight limits of 17.4/14.4 being significantly smaller than and incompatible with the recommended BMI values.
\end{abstract}

\section{Introduction}

Overweight and obesity are serious health problems $[1,2]$ which have increased during the past decades $[1,3]$ in the US, in other "Western" countries [4], and globally [5]. They tend to cause health damages with potentially immense expenditures $[5,6]$. An appropriate definition of overweight and obesity is essential to the Public Health community for designing and monitoring interventions.

Overt obesity can be recognized without measurement. However, proper assessment of moderate overweight is essential to targeting "action" thresholds. Currently, the Body Mass Index BMI is used for defining overweight and obesity. The BMI, given in units $\mathrm{kg} / \mathrm{m}^{2}$, has the dimension of an area density, the average value of which, for normal, healthy subjects, has so far been recommended as $21.7[2,6]$. In the past, also the Broca formula [7] was used, a linear mass versus height relationship, which implies a linear density $(\mathrm{kg} / \mathrm{m})$, which may be relevant for describing a hair, but not a human body. The BMI has been recommended for use among all age groups from childhood through adulthood [2]. Analyzing the relationship between BMI and adiposity, Revicki and Israel state "... there exists considerable error associated with the prediction of body fat, using different BMIs" [8]. Overweight is defined by a BMI between 25.0 and 29.9 [2] and underweight by a BMI below 18.5. People with BMI greater than 30.0 are categorized as obese. "Such terminology is useful... yet remains imperfect" [2]. As "... many physicians and patients find it" (the BMI) "difficult to interpret" [3], a useful body mass relationship, indeed, should have dimensions relevant to the human body, a volume density or pressure. The BMI has been used for more than three decades by now, but the fraction of overweight and adipose people is still increasing dramatically. This may be due to the fact that people just do not care. Moreover, the BMI formula may be inapplicable for all individuals from birth to old ages. Thirdly, the recommended values may require adjustment. This is the central issue of this study. In 


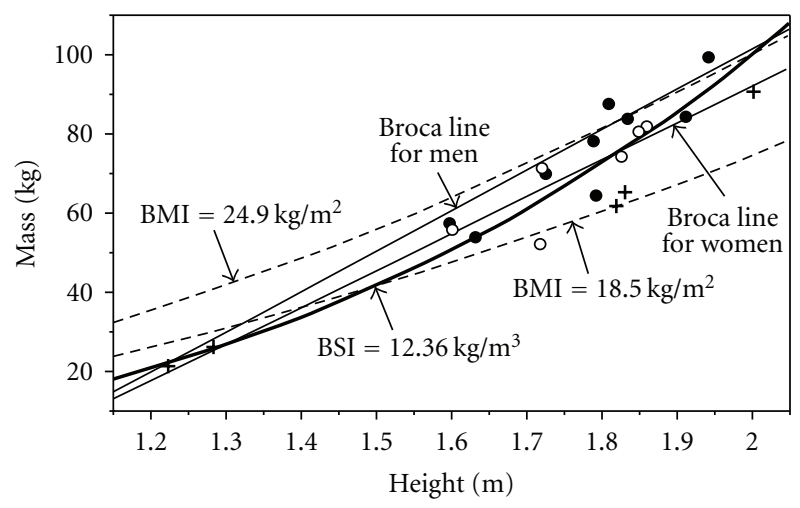

Each point gives data from an individual person.

+ Persons up to 32 years

- Persons between 36 and 57 years

- Persons older than 58 years

FIGURE 1

order to clarify these issues, this paper examines five cohorts with regard to both BMI and BSI. These cohorts are (a) individuals between 8 years and 76 years of age, mainly adults and (b) four age cohorts of children from birth to the age of 14.5 years. The results indicate on the one hand that the BMI is less accurate than the BSI in individuals older than $\sim 8$ years and taller than $\sim 1.3 \mathrm{~m}$. On the other hand, the BMI is more accurate than the BSI for children below the age of $\sim 8$ years and shorter than $\sim 1.3 \mathrm{~m}$ (BMI underweight limit 14.4, overweight limit 17.4). These BMI values for children are significantly lower than the conventionally used BMI given above.

\section{Methods}

2.1. Units and Dimensions. Our approach is based on a careful use of units and dimensions. International standards require that masses are measured in kilogram $(\mathrm{kg})$, lengths or heights in meter $(\mathrm{m})$, and forces or weights in Newton $(\mathrm{N})$. The $\mathrm{kg}$ has been used also for weights. To avoid confusion, the $\mathrm{kg}$ weight was termed "kilopond" $(=9.80665 \mathrm{~N})$ [9]. "M" in BMI implies a mass. Thus, the BMI clearly has the dimension of an area density, which is common, for instance, in characterizing paper. When interpreting the "BMI" not as Body Mass Index but as Body Weight Index "BWI", its dimension becomes weight/area, which is "pressure" and that is relevant to the human body. Since the BMI is numerically equal to the BWI, we use the BMI in this paper to be synonymous with "BWI", unless stated otherwise.

\subsection{Relationship Body Mass to Body Height, BMI versus BSI.} We also refer to the fact that humans can swim. With airfilled lungs humans can float because the average body density is slightly less than the density of water. Without air in the lungs people drown. The body density varies during breathing by about $\pm 2-3 \%$. Without considering anatomical details, the average human body density is $\sim 1000 \mathrm{~kg} / \mathrm{m}^{3}$.

For finding an appropriate relationship between body mass $\mathrm{w}$ and body height $\mathrm{H}$, we apply the model of scaling, the elementary rule of magnification of a three-dimensional body: if the height of a body-for example, a sculpture-is changed by a factor of $x$, then also its width and thickness must change by the same factor. Because of the nearly constant density of the human body, this immediately leads to the relation $w / H^{3}=$ constant, with the dimension $\mathrm{kg} / \mathrm{m}^{3}$. We set $w / H^{3}=$ BSI [10]. Similarly $w / H^{2}=$ BMI. The BSI expresses a volume density that is relevant to the human body. The exponent (3) is mandatory for similar threedimensional shapes. If shapes change, as in children developing from babies to adults, the scaling model is no longer valid. Therefore, the BSI obtained in adults and recommended to describe the normal mass-versus-height relationship is expected to mislead when applied to younger children.

The meaning of other exponents (like 2 in the BMI or 1 for the Broca formula) must be questioned. For these exponents, there exists not even a theoretical model but only an arbitrary advance, unless one uses the BWI, as explained above. Because of the complexity of the human body, one should not expect that a simple approach, like with the BSI or the BMI as single parameters (like 21.7 for the BMI, and 12.36 for the BSI) is able to describe accurately the relationship between body mass and body height from birth until death [11]. Both the BMI and BSI approaches are valid only approximately. A useful body mass versus body height relationship should be not only simple but also allow for differences by age, gender, and ethnicity. This study, thus, presents a critical and numerically careful test of the usefulness of the BMI and the BSI (models) in order to determine the relevant parameters and their uncertainties in cohorts of normal weight individuals.

The body height can be measured with a precision of better than $0.2 \%$ and the body mass to less than $1 \%$. This is much better than needed, because the BMI values, for instance, for healthy [2] or normal weight [6] people range between 18.5 and 24.9 , with the average of $21.7 \pm 15 \%$.

2.3. Data Acquisition and Analysis. Where scaling does not apply, for instance, for small children, we have used a sufficiently large sample of healthy individuals and measured their heights and weights in order to obtain an adequate data base for our analysis. As people are not equal genetically or in their environmental situations and as considerable differences exist regarding their bodily proportions, one expects a cloud of points in the mass versus height diagram (Figure 1). The task here is then to determine how the BMI and BSI approaches mathematically fit [12] the individual data points. For that purpose, we define the BMI value for each individual as $a_{M i}=w_{i} / H_{i}{ }^{2}$ and the corresponding BSI values as $a_{S i}=w_{i} / H_{i}{ }^{3}$. Then we apply a least squares fit [12] to all data points, both for $a_{M i}$ and $a_{S i}$. We use masses and body heights of individuals (Tables 1,2, and 3) for comparing BMI with BSI. For such tasks, careful data handling and error analysis are required. Decades ago investigators restricted their analyses to average heights and weights because of the ease of calculation [13].

Flegal [13] importantly noted the difference between younger and older men and women, as was also emphasized by Shreeve and Pierson [14]. In view of the natural changes of normal bodies with growing and ageing (healthy young 
TABle 1: Gender, heights $H$, and masses $w$ of the people, whose data are plotted as points in Figure 1. Individuals are listed in the sequence of observations made.

\begin{tabular}{lccc}
\hline Gender & Height $H(\mathrm{~m})$ & Mass $w(\mathrm{~kg})$ & Age (years) \\
\hline \multirow{6}{*}{ Male } & 1.81 & 87 & 73 \\
& 1.91 & 83 & 59 \\
& 1.94 & 98 & 71 \\
& 1.86 & 81 & 44 \\
& 1.72 & 69 & 70 \\
& 1.83 & 73 & 36 \\
& 1.85 & 80 & 53 \\
& 1.83 & 83 & 65 \\
& 2.00 & 89 & 32 \\
Female & 1.79 & 76 & 69 \\
& 1.79 & 64 & 76 \\
\hline & 1.63 & 53 & 65 \\
& 1.71 & 51 & 57 \\
& 1.29 & 26 & 10 \\
& 1.22 & 21 & 8 \\
& 1.60 & 55 & 40 \\
& 1.60 & 57 & 62 \\
& 1.72 & 70 & 54 \\
& 1.83 & 64 & 28 \\
& 1.82 & 61 & 22 \\
\hline
\end{tabular}

subjects usually have less body fat than healthy older subjects), it seems appropriate to follow Flegal [13]. We propose to extend this approach to observe the effects of age and gender, of ethnic, genetic, and other distinctions on the BSI and BMI values. This task requires a worldwide effort probably taking a decade or more. The emphasis of this paper is to clarify the applicability of the BMI and BSI and thus to provide a solid bases for these studies.

\section{Results}

3.1. Normal Individuals, BMI, and BSI. In a first step, we have taken data of 20 healthy and normal weight people listed in Table 1, all ethnically Caucasions. The heavy line in Figure 1 is the BSI curve for these people. The two dashed lines depict the $18.5 \mathrm{~kg} / \mathrm{m}^{2}$ and $24.9 \mathrm{~kg} / \mathrm{m}^{2}$ BMI curves, the lower and upper limits for normal as determined by others $[2,6]$. In addition, the Broca lines are given for men and women. For the 20 healthy people, data points relate individual masses in terms of weights and heights, as listed in Table 1 . The crosses identify people from 8 to 32 years, open circles people between 36 and 57 years, and full circles people from 58 to 76 years. The total cohort shows a scatter typical for "normal" people. This scatter mainly expresses the differences of people's bodies. The measurement errors regarding weights and height are insignificant in this context. The scatter also explains the difficulty of finding a "correct" relationship between body height and body mass. The crosses, open circles, and full circles even provide evidence for an age dependence of the human weight in support of Flegal [13] and Shreeve and Pierson [14]. Figure 1 illustrates that a linear fit cannot cover adequately all data points. A linear fit tends to make both tall and short people appear overweight. Moreover, people who have masses in agreement with the BSI appear BMIoverweight if they are tall and BMI-underweight if they are short. This results from the mathematical differences in slopes of the BMI and BSI curves.

3.2. "Precision" Comparison between BMI and BSI in General. To find out whether and where BSI or BMI is superior for classifying the relationship between observed weights and heights of a group of individuals, we have used the individual values of the 20 healthy people listed in Table 1 to calculate each individual BMI and BSI. As introduced above, we express the individual BMI by the symbol $a_{M i}=w_{i} /\left(H_{i}{ }^{2}\right)$ and the individual BSI by the symbol $a_{S i}=w_{i} /\left(H_{i}{ }^{3}\right)$, with the subscript i specifying the individual. With standard error analyses [12], we have obtained the mean values $a_{S}$ and $a_{M}$. The advantage of the standard error analysis is that it provides the standard deviations (uncertainties) $\sigma\left(a_{S}\right)$ and $\sigma\left(a_{M}\right)$ of $a_{S}$ and $a_{M}$, and also the mean uncertainties $\sigma\left(a_{S i}\right)$ and $\sigma\left(a_{M i}\right)$ for the scatter of the individual data points. We list only the numerical values, omitting the dimensions, and obtain:

$$
\begin{array}{rrr}
a_{S}=12.36, \quad \sigma\left(a_{S}\right) & =0.30, \quad \sigma\left(a_{S i}\right)=1.4, \\
a_{M}=21.48, & \sigma\left(a_{M}\right)=0.74, & \sigma\left(a_{M i}\right)=3.3 .
\end{array}
$$

$\sigma\left(a_{S}\right)$ is $2.4 \%$ of $a_{S}$, and $\sigma\left(a_{M}\right)=3.4 \%$ of $a_{M}$. For $a_{M}$ we obtained 21.48 , which is only $1 \%$ and thus insignificantly smaller than the average BMI value of $21.7[2,6]$. This shows that our sample average is in very good agreement with the BMI average, determined by others $[2,6]$.

Even our small sample indicates that the BSI approach yields standard deviations which are a factor of $\sim 1.4$ smaller than the BMI approach. The scattering of the data clouds $\left(\sigma\left(a_{S i}\right)\right.$ and $\left.\sigma\left(a_{M i}\right)\right)$ is $11 \%(=1.4 / 12.36)$ and $15 \%$ $(=3.3 / 21.48)$, respectively. The latter corresponds extremely well with the distance of $15 \%$ of the BMI curves 18.5 and 24.9 from the mean at 21.7. However, the $11 \%$ suggest a more narrow range, for the BSI $(15 \% / 11 \%=1.4)$. Thus, the BSI model fits our data set (Table 1) better than the BMI.

One may ask whether a sample size of only $n=20$ individuals is acceptable for assessing BMI and BSI. Answer 1 is that the "precision" $\sigma\left(a_{S}\right)$ is proportional to $n^{-0.5}$. Therefore, a factor 10 improvement in "precision" would have required a hundred times larger sample size, in our case 2000. Answer 2 is that the scattering of the individual data, $\sigma\left(a_{S i}\right)$, is practically independent of the sample size. Therefore, a sample size of 20 is sufficient for discriminating between BMI and BSI.

The data in Figure 1 already show an age dependence. Selecting the five youngest people in this group (symbol $Y$ ), we find

$$
a_{S, Y}=11.06, \quad \sigma\left(a_{S, Y}\right)=0.39, \quad \sigma\left(a_{S, Y i}\right)=0.9 .
$$


The difference between $a_{S, Y}=11.06$ and $a_{S}=12.36$, as given above, is -1.3 and significant. We find correspondingly for the 9 seniors (symbol $A$ ):

$$
a_{S, A}=13.05, \quad \sigma\left(a_{S, A}\right)=0.38, \quad \sigma\left(a_{S, A i}\right)=1.1 .
$$

Here the difference between $a_{S, A}(4)$ and $a_{S, Y}(3)$ is $\sim 2.0$ and amounts to 3.7 standard deviations. This is sufficient to establish the age dependence for the BSI.

We also compute the analogous values for the BMI and obtain for the five younger people:

$$
a_{M, Y}=17.87, \quad \sigma\left(a_{M, Y}\right)=1.4, \quad \sigma\left(a_{M, Y i}\right)=3.2 .
$$

It is worth noting that $a_{M, Y}$ is lower than the BMI value of 18.5 , the limit to BMI-underweight.

For the 9 seniors, we find correspondingly

$$
a_{M, A}=23.24, \quad \sigma\left(a_{M, A}\right)=0.79, \quad \sigma\left(a_{M, A i}\right)=2.4 .
$$

The difference between $a_{M, A}$ (6) and $a_{M, Y}$ (5), that is, between 23.24 and 17.87 is $~ 5.4$. This amounts to 3.3 standard deviations. Thus, also the BMI approach shows clearly an age dependence.

\subsection{The Cohort of Children}

3.3.1. Total Cohort. For a more detailed study of the body mass relationships, we have analysed in addition data of 79 children, 42 girls (Table 2) and 37 boys (Table 3 ). These data have been determined by one of us (SZ) during routine preventive medical checkups of healthy children with normal growth not selected for their weights, most of which appeared normal. Figure 2 shows the data points for the girls $(\mathrm{x})$ and boys $(\bullet)$. Independently shown are the two conventional BMI curves of 18.5 for underweight and 24.9 for overweight, and the BSI curve of 12.36 .

3.3.2. Cohorts of 4 Age Groups, BMI, and BSI. For the analysis, we have split the data of the total cohort into 4 age cohorts of both the girls and boys, with data listed in Tables 2 and 3 , and obtained the following results:

Cohort 1. Age group from birth-1.0 year

$$
\begin{array}{cccc}
\text { Girls: } & a_{M}=15.1, & \sigma\left(a_{M}\right)=0.4, & \sigma\left(a_{M i}\right)=1.4, \\
& a_{S}=24.0, & \sigma\left(a_{S}\right)=0.9, & \sigma\left(a_{S i}\right)=3.3 . \\
\text { Boys: } & a_{M}=16.9, & \sigma\left(a_{M}\right)=0.5, & \sigma\left(a_{M i}\right)=1.8, \\
& a_{S}=26.0, & \sigma\left(a_{S}\right)=0.9, & \sigma\left(a_{S i}\right)=3.0 .
\end{array}
$$

\begin{tabular}{|c|c|c|c|}
\hline Nr. & Height $\mathrm{H} / \mathrm{m}$ & Mass w/kg & Age/years \\
\hline 1 & 1.46 & 34.0 & 8.0 \\
\hline 2 & .85 & 11.1 & 2.0 \\
\hline 3 & 1.02 & 14.6 & 3.1 \\
\hline 4 & .97 & 15.8 & 3.3 \\
\hline 5 & .95 & 13.4 & 2.1 \\
\hline 6 & 1.06 & 17.6 & 4.0 \\
\hline 7 & 1.11 & 21.3 & 3.1 \\
\hline 8 & .85 & 12.0 & 2.0 \\
\hline 9 & 1.05 & 17.6 & 5.0 \\
\hline 10 & .98 & 15.5 & 5.0 \\
\hline 11 & 1.28 & 28.1 & 9.0 \\
\hline 12 & .88 & 12.0 & 2.0 \\
\hline 13 & 1.76 & 60.6 & 14.1 \\
\hline 14 & 1.44 & 41.0 & 10.0 \\
\hline 15 & 1.64 & 59.0 & 14.0 \\
\hline 16 & 1.27 & 25.8 & 7.1 \\
\hline 17 & 1.28 & 25.0 & 8.0 \\
\hline 18 & 1.31 & 44.5 & 8.0 \\
\hline 19 & 1.55 & 43.0 & 13.3 \\
\hline 20 & 1.66 & 66.6 & 14.1 \\
\hline 21 & .50 & 3.5 & Birth \\
\hline 22 & 1.03 & 16.0 & 3.0 \\
\hline 23 & .48 & 3.16 & Birth \\
\hline 24 & .54 & 3.54 & Birth \\
\hline 25 & .77 & 8.1 & 1.0 \\
\hline 26 & 1.57 & 51.8 & 13.5 \\
\hline 27 & 1.58 & 52.7 & 14.5 \\
\hline 28 & .63 & 6.73 & 0.4 \\
\hline 29 & .75 & 8.55 & 1.0 \\
\hline 30 & .62 & 5.93 & 0.4 \\
\hline 31 & .65 & 6.91 & 0.4 \\
\hline 32 & .77 & 9.06 & 0.83 \\
\hline 33 & .70 & 7.7 & 0.83 \\
\hline 34 & 1.78 & 46.0 & 13.7 \\
\hline 35 & 1.74 & 40.0 & 13.7 \\
\hline 36 & .72 & 8.11 & 0.83 \\
\hline 37 & .78 & 9.55 & 0.83 \\
\hline 38 & 1.58 & 59.0 & 12.2 \\
\hline 39 & .62 & 6.01 & 0.3 \\
\hline 40 & 1.62 & 58.9 & 13.1 \\
\hline 41 & 1.33 & 34.6 & 8.7 \\
\hline 42 & 60 & 5.45 & 0.3 \\
\hline
\end{tabular}

TABLE 2: Data of girls from birth until 14.5 years. 
TABLE 3: Data for boys from birth until 14 years.

\begin{tabular}{|c|c|c|c|}
\hline Nr. & Height H/m & Mass w/kg & Age/years \\
\hline 1 & .99 & 13.9 & 1.11 \\
\hline 2 & .84 & 10.8 & 1.11 \\
\hline 3 & 1.38 & 29.8 & 10 \\
\hline 4 & 1.73 & 57.1 & 14.0 \\
\hline 5 & 1.80 & 75.1 & 14.0 \\
\hline 6 & 1.38 & 35.0 & 8.0 \\
\hline 7 & .99 & 14.6 & 3.1 \\
\hline 8 & .89 & 12.0 & 1.11 \\
\hline 9 & .98 & 16.9 & 2.11 \\
\hline 10 & .86 & 12.6 & 1.11 \\
\hline 11 & 1.04 & 18.5 & 5.4 \\
\hline 12 & 1.38 & 30.2 & 8.0 \\
\hline 13 & 1.39 & 32.6 & 10.0 \\
\hline 14 & 1.01 & 17.5 & 3.10 \\
\hline 15 & 1.04 & 15.9 & 4.0 \\
\hline 16 & 1.12 & 17.8 & 5.2 \\
\hline 17 & 1.02 & 16.0 & 2.11 \\
\hline 18 & 1.10 & 18.5 & 3.11 \\
\hline 19 & .92 & 15.0 & 2.0 \\
\hline 20 & 1.04 & 17.4 & 2.11 \\
\hline 21 & .53 & 3.6 & Birth \\
\hline 22 & 1.13 & 17.5 & 5.0 \\
\hline 23 & .79 & 10.9 & 0.92 \\
\hline 24 & .69 & 7.34 & 0.75 \\
\hline 25 & 1.43 & 42.7 & 9.3 \\
\hline 26 & .73 & 9.64 & 0.83 \\
\hline 27 & .64 & 6.89 & 0.25 \\
\hline 28 & .74 & 10.95 & 1.0 \\
\hline 29 & .68 & 7.8 & 0.25 \\
\hline 30 & .65 & 7.47 & 0.33 \\
\hline 31 & .65 & 7.55 & 0.33 \\
\hline 32 & .80 & 11.1 & 0.83 \\
\hline 33 & .60 & 5.86 & 0.25 \\
\hline 34 & .47 & 3.35 & 0.27 \\
\hline 35 & 1.38 & 34.0 & 9.6 \\
\hline 36 & 1.41 & 29.5 & 9.6 \\
\hline 37 & .59 & 5.93 & 0.25 \\
\hline
\end{tabular}

Cohort 2. Age group above 1 year-3.0 years

$$
\begin{array}{llll}
\text { Girls: } & a_{M}=15.6, & \sigma\left(a_{M}\right)=0.4, & \sigma\left(a_{M i}\right)=0.8, \\
& a_{S}=17.3, & \sigma\left(a_{S}\right)=1.0, & \sigma\left(a_{S i}\right)=2.2 . \\
\text { Boys: } & a_{M}=16.1, & \sigma\left(a_{M}\right)=0.5, & \sigma\left(a_{M i}\right)=1.3, \\
& a_{S}=17.2, & \sigma\left(a_{S}\right)=0.8, & \sigma\left(a_{S i}\right)=2.1 .
\end{array}
$$

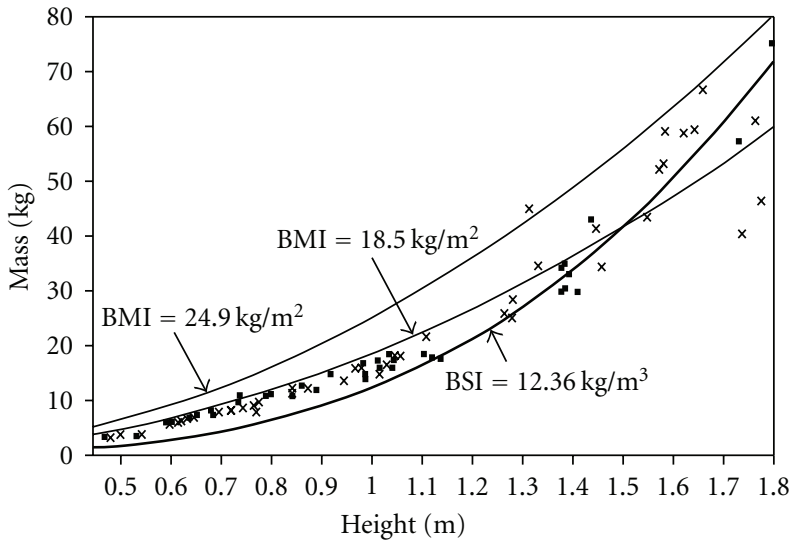

Each point gives data from an individual person.

$\times$ For girls

- For boys

FIGURE 2

Cohort 3. Age group 3.1 years-7.1 years

Girl

$$
\begin{array}{llcc}
\text { Girls: } & a_{M}=16.1, & \sigma\left(a_{M}\right)=0.4, & \sigma\left(a_{M i}\right)=1.0, \\
& a_{S}=15.2, & \sigma\left(a_{S}\right)=0.6, & \sigma\left(a_{S i}\right)=1.6 . \\
\text { Boys: } & a_{M}=15.3, & \sigma\left(a_{M}\right)=0.6, & \sigma\left(a_{M i}\right)=1.4, \\
& a_{S}=14.5, & \sigma\left(a_{S}\right)=0.7, & \sigma\left(a_{S i}\right)=1.9 .
\end{array}
$$

Cohort 4. Age group 8 years-14.5 years

$$
\begin{array}{cccc}
\text { Girls: } & a_{M}=19.6, & \sigma\left(a_{M}\right)=1.0, & \sigma\left(a_{M i}\right)=3.7, \\
& a_{S}=12.9, & \sigma\left(a_{S}\right)=0.8, & \sigma\left(a_{S i}\right)=2.9 . \\
\text { Boys: } & a_{M}=18.1, & \sigma\left(a_{M}\right)=0.9, & \sigma\left(a_{M i}\right)=2.7, \\
& a_{S}=12.3, & \sigma\left(a_{S}\right)=0.5, & \sigma\left(a_{S i}\right)=1.3 .
\end{array}
$$

The BMI analysis for children in the youngest three age cohorts (cohort 1-3) yields $a_{M}$ values which are consistent with a single number of $\sim 15.9$ both for girls and boys. As the children become older (cohort 4), the $a_{M}$ for girls rises to 19.6 and for boys to 18.1 . These $a_{M}$ values are still significantly below the conventional average BMI value of 21.7.

However, regarding the BSI analysis in children of the first cohort, up to one year, the as was found to be 24.0 for girls and 26.0 for boys, which is much higher than the BSI for adults (12.36). As the children grow older (see cohorts 2), the as value decreases to $\sim 17.3$, being about equal for girls and boys. In the cohort 3 , children with ages between $\sim 3$ to $\sim 7$ years have an as of 15.2 for girls and 14.5 for boys. However, the as in children of cohort 4 , ageing between $\sim 8$ and 14.5 years, is 12.9 for the girls and 12.3 for the boys. These results agree with the BSI value of 12.36 that has been found for healthy weight adults. This shows that the BSI based on scaling (magnification) underestimates the normal 
weights of small children. This underestimation disappears as the children reach ages above $\sim 8$ years. The reason for this is easily explained by the differences in the body proportions typically for these children compared to those of adults.

3.4. Precision Comparison, BMI, and BSI. The results of our analysis, separately for girls and boys, clearly show that for children older than $\sim 8$ years or taller than $\sim 1.3 \mathrm{~m}$, the BSI is preferable to the BMI for classifying normal and abnormal weights of children. The ratios of $\sigma_{S} / \sigma_{M}$ and $\sigma_{S i} / \sigma_{M i}$, as given above, are on the average $1 / 1.6$, which implies lower ranges of uncertainties with BSI and thus its higher precision.

For the children of cohort 4, that is, 25 girls and boys older than $\sim 8$ years and taller than $\sim 1.3 \mathrm{~m}$, we obtain for the BSI:

$$
a_{S}=12.70, \quad \sigma\left(a_{S}\right)=0.47, \quad \sigma\left(a_{S i}\right)=2.3 .
$$

The $a_{S}$-value, 12.70, differs from 12.36 (adult BSI) by 0.34, which is only 60 per cent of the combined errors of the BSI values of 12.70 and 12.36 . This means that the $a_{S}$ value of all 25 children older than $\sim 8$ years and taller than $\sim 1.3 \mathrm{~m}$ agrees well with the reference BSI value of 12.36 for adults and is again indicative of the higher precision of the BSI compared with the BMI in this age group.

However, for the individual cohorts 1-3, children younger than $\sim 8$ years or shorter than $\sim 1.3$, we have listed above the $a_{M}$ values, which are close to $\sim 15.9$. The standard error analysis of the whole group of 54 girls and boys (cohorts 1-3) yields for the BMI:

$$
a_{M}=15.9, \quad \sigma\left(a_{M}\right)=0.20, \quad \sigma\left(a_{M i}\right)=1.5 .
$$

The $a_{M}$-value of 15.9 , being the average for the cohorts $1-3$ with a total of 54 children, differs from the conventionally used BMI average of 21.7. This difference of 5.8 corresponds with 29 standard deviations of $\sigma\left(a_{M}\right)=0.20$ (see above), the most significant discrepancy between the BMI here found for children and the conventionally used BMI.

The above equation (12) with $a_{M}$ of 15.9 and $\sigma\left(a_{M i}\right)$ of 1.5 implies over- and underweight limits of 17.4 and 14.4, respectively, and relative to 15.9 , a precision of $9.4 \%$, in contrast to the conventional BMI which has a precision of $\sim 15 \%$. Note that over- and underweight limits are defined here as $a_{M} \pm \sigma\left(a_{M i}\right)$.

Therefore, healthy short people would be lighter than what the conventional BMI suggests. This agrees with what we find for people older than 8 years using the BSI, as discussed above.

As the BMI has been recommended for use among all age groups from childhood through adulthood [2] and as our children are "normal", the BMI values, in particular the BMI- underweight value of 18.5, should be revised and not be applied for children below $\sim 8$ years of age. For children older than 8 years, the BMI should be replaced by the BSI.

The results, in summary, show two relevant facts.

(1) The conventional BMI appears less precise than the BSI for adults and individuals older than 8 years and should accordingly be replaced.
(2) The BMI applies well for children younger than 8 years or shorter than $1.3 \mathrm{~m}$ provided it's average is reduced from the conventional value of 21.7 to 15.9 with underweight and overweight limits being 14.4 and 17.4, respectively.

\section{Discussion}

4.1. BMI and BSI Relevance in Adults and Children of Different Age Groups. Applying standard data analysis [12] on our first sample of 20, mainly adult healthy individuals, we have observed that the BSI body mass equation yields parameters that are a factor of 1.4 more precise than those for the BMI. Both BMI and BSI analyses disclose age dependence, even for our small sample. The BMI analysis leads to an average body mass index (21.48) for our sample in excellent agreement with the BMI average of 21.7. This shows that the choice of our sample (Table 1) was unbiased. Our analysis of the data (Tables 2 and 3) on children demonstrates that the overwhelming part of them is BMI underweight. This casts serious doubt on the usefulness of the BMI-underweight limit if applied to children. For very young children, caution should be exercised when using the BSI because the body shape of these children cannot be reproduced by scaling shapes of adults down to these children. For children older than $\sim 8$ years and taller than $\sim 1.3 \mathrm{~m}$, the BSI values for girls and boys are in good agreement with 12.36, the BSI value of the sample cohort with data listed in Table 1.

Our analysis of 54 children with ages less than $\sim 8$ years or shorter than $\sim 1.3 \mathrm{~m}$ shows that the BMI is the adequate body mass relationship with an average BMI value of 15.9, albeit different from the corresponding BMI for adults. Moreover, the number 15.9 is less than the BMI-underweight limit [2]. The analysis of the children data yields a BMIunderweight limit of 14.4. As a first reasonable estimate, we define underweight if the BMI value of a child is more than $\sigma\left(a_{M i}\right)=1.5$ less than 15.9, which means: below BMI $=14.4$. We observe that 9 of our 79 children, 5 girls and 4 boys, are in the category of underweight, $\sim$ one sixth of them are in agreement with expectation for a normal statistical distribution. Similarly, we define overweight if the individual BMI value is more than $\sigma\left(a_{M i}\right)$, above 15.9 . This would be the case for children with BMI above 17.4. Seven children, $\sim 13$ percent, only boys, fall into that category. Interestingly, this would imply that we should consider children already as overweight if they are even 1.1 units below the standard BMI-underweight limit of 18.5. This obvious and serious discrepancy needs correction perhaps by revising the BMI recommendation [2] in order to differentiate normal values for short or very young children from values for older, taller children plus adults.

There exist slide-rule-type circular plastic discs "EXFORGE", where body height and body weight can be matched by rotating one disc relative to the other, and then the BMI value can be read off. In a little window, the border to underweight is shown as 18.5. Our data on children show that this disc leads to incorrect conclusions.

The study of Danish school children with 7 through 13 years of age by Baker et al. [15] has shown that higher 
BMI during childhood is associated with an increased risk of coronary heart disease in adulthood. The very large sample size in the Danish study ensures that these data are of high statistical relevance. Table 1 of the publication by Baker et al. shows fourteen BMI values between 15.4 and 18.6 that are, with a single exception (18.6) lower than 18.5, below which people are judged underweight, if one adopts the recommendation for use among all age groups from childhood through adulthood [2]. The BMI values of our children (cohorts 1-3) are fully consistent with the data given by Baker et al. in Table 1 of their paper.

Furthermore, we find that the weights and heights of our children from 8-14.5 years can be well described by the BSI model that fits the data of adults better than the BMI does. Our study provides both BMI and BSI fits for children from birth up to an age of $\sim 8$ years. Shorter children may appear of normal or even subnormal weight when using the BMI as recommended so far [2], but they actually may be overweight as we have discussed above on the basis of our BMI analysis. Using the BMI with the "underweight limit" of 18.5 as the only reference may influence parents of children with normal weight (according to our study) to feed them until they have "normal weight" with BMI $=21.7$, but actually then they are overweight. An essential question is which BMI/BSI values signal a significant risk of a given age group not only for cardiovascular diseases in adulthood but also of other diseases associated with overweight. This paper provides the answer. Obviously, the most appropriate tools needed to be applied, BMI and BSI, at the appropriate ages and heights for correct prognostic values.

The BSI formula is identical with that known as Rohrer's "Ponderal Index" (PI) [16-18] used to evaluate perinatal problems in new-born infants or near-term foetuses who are small for gestational age, or who have intrauterine growth retardation. The PI has been shown to be better than birth weight for prediction of short-term problems such as neonatal asphyxia, acidosis, hypoglycaemia, and hypothermia [18]. Other studies [19, 20] have used the PI to predict long-term comorbidities such as microalbuminuria, insulin resistance, hypertension, and other cardiovascular diseases. Obviously, the BMI value for children, as shown above, supersedes the precision of the PI.

The individually measured BMIs, interpreted as body weight indices, are proportional to the pressure in all weightbearing joints. When people with identical heights are compared, their joint pressures are proportional to their BMIs, or simply to their masses or weights. The lighter person at a given height has an advantage. Individuals with the same BSI have pressures in their weight-bearing joints proportional to their heights. Therefore, the shorter person who is lighter for a given BSI has an advantage.

4.2. Proposal for Large Scale Studies. The studies of Bhargava et al. [21] suggest that it would be valuable to measure the BMI for sufficiently many people, males and females, from birth on, preferably annually and in various regions all over the world. The present study shows that the BSI is more precise than the BMI for people older than 8 years or taller than $\sim 1.3 \mathrm{~m}$. Our corresponding average
BSI value is 12.5 with a limit to underweight around 11.1 and the limit to overweight around 13.9. For children with ages less than $\sim 8$ years or shorter than $\sim 1.3 \mathrm{~m}$, we find the BMI to be superior to the BSI, if the conventional BMI value of 21.7 is replaced by 15.9 as average value, bringing a reduction by 27 percent. For improving the $a_{M^{-}}$and $a_{S}$-values and finding better underweight and overweight limits for BMI and BSI we strongly recommend a worldwide study of many subgroups for which individual "best" values should be determined. We propose that such studies evaluate the BMI and the BSI metric for achieving a higher predictive power for genetic and other variations in different world regions, consequential ranges of metabolic differences, and possible environmental effects. Such a global investigation could provide the most useful information about normal metabolism, the epidemiology of adiposity under the multiple and interacting influences of nutrition, exercise, race, and other measurable factors. It would be interesting to include the BSI together with the BMI in the BODE index [22].

4.3. Outlook. For this future work, we therefore propose to study large samples, of healthy people in age categories, irrespective of height, separately for males and females. Additionally, one should simultaneously consider racial/ethnic and other distinctions and measure their weights and heights; also the blood pressure appears of interest in this context. If scaling would apply also to human vascular system, one could directly show the proportionality between BSI and blood pressure [9] using the Hagen-Poiseuille-Law. Overall, in doing so one may easily have to deal with 300 or more subgroups “ $j$ ”. For each group with preferably more than 2000 individuals, the fit [12] will allow to find the mean numbers $a_{S j}$, the numerical BSI values for each group $j$. Similarly numerical values should be obtained for the BMIs. As the fits also will yield the standard errors $\sigma$ of the $a_{S j^{-}}$ and $a_{M j}$-values, the changes with age for each gender can be described quantitatively. Also the effects of ethnic and other distinctions could then be disclosed. The results of such future studies should allow setting narrower and more accurate definitions for healthy weight than those presently achieved by the exclusive use of the BMI. With the BSI values, it should be possible to quantify overweight or adiposity more precisely than with the BMI alone, in people older than $\sim 8$ years or taller than $1.3 \mathrm{~m}$. Based on these $a$-values taken only for healthy weight, one should then consider all people, healthy and ill, assign each person to the relevant subgroup " $j$ ", and record, in addition to the weight and height of each person, clinical data including information about food intake in order to link a person's body mass data with his or her clinical status. This is clearly a task for future decades.

\subsection{Suggestion for Normal Weights versus Heights in Medical} Practice. For medical practice, Table 4 gives healthy weights calculated with the BSI formula $w=$ BSI $\cdot H^{3}=12.5 \mathrm{~kg} / \mathrm{m}^{3}$. $H^{3}$ for ages above $\sim 8$ years and heights above $\sim 1.3 \mathrm{~m}$, and with the BMI formula $w=\mathrm{BMI} \cdot H^{2}=15.9 \mathrm{~kg} / \mathrm{m}^{2} \cdot H^{2}$ for ages less than 8 years and heights below $1.3 \mathrm{~m}$. For heights 
TABLE 4: Healthy weights for people with heights of $1.3 \mathrm{~m}$ and above obtained with the BSI formula using $a_{S}=12.5 \mathrm{~kg} / \mathrm{m}^{3}$. For individuals shorter than $1.3 \mathrm{~m}$ the BMI has been used with $a_{M}=$ $15.9 \mathrm{~kg} / \mathrm{m}^{2}$. Mass ranges are based on the individual $\sigma\left(a_{M i}\right)$ values for heights below $1.3 \mathrm{~m}$, and on $\sigma\left(a_{S i}\right)$ values for heights above $1.3 \mathrm{~m}$ taken from (1) and (12). In column 1 are listed the heights $H_{i}$ of the people (in meters), in column 2 are given the ideal healthy weights or masses $w_{i}$ (in $\mathrm{kg}$ ) and in column 3 are listed individual healthy weight ranges (also in $\mathrm{kg}$ ). For heights between the listed values an interpolation between the neighbouring weights and weight ranges should be sufficient.

\begin{tabular}{lcc}
\hline Height $\mathrm{H}_{\mathrm{i}} / \mathrm{m}$ & Mass $\mathrm{w}_{\mathrm{i}} / \mathrm{kg}$ & $\begin{array}{c}\text { Healthy weight } \\
\text { Mass range/kg }\end{array}$ \\
\hline 0.5 & 4.0 & $3.6-4.4$ \\
0.6 & 5.7 & $5.2-6.3$ \\
0.7 & 7.8 & $7.1-8.5$ \\
0.8 & 10 & $9-11$ \\
0.9 & 13 & $12-14$ \\
1.0 & 16 & $14-18$ \\
1.1 & 19 & $17-21$ \\
1.2 & 23 & $21-25$ \\
1.3 & 27 & $24-30$ \\
1.4 & 34 & $30-38$ \\
1.5 & 42 & $37-46$ \\
1.6 & 51 & $45-56$ \\
1.7 & 61 & $54-67$ \\
1.8 & 72 & $64-80$ \\
1.9 & 85 & $75-94$ \\
2.0 & 99 & $88-110$ \\
2.1 & 114 & $102-127$ \\
2.2 & 132 & $117-146$ \\
\hline
\end{tabular}

between the tabulated values $H_{i}$ and $H_{i+1}$, interpolation between the weights $w_{i}$ and $w_{i+1}$ is sufficiently precise. In the future, the list should be replaced by improved data for the individual subgroups “ $j$ ”.

\section{Conclusion}

In conclusion we find that the BSI with a mean of $\sim 12.5$ is a dimensionally correct body mass relationship for people older than $\sim 8$ years or taller than $\sim 1.3 \mathrm{~m}$ and has a higher precision than the BMI. If understood as body weight index (BWI) with the dimension of weight/area, the BMI expresses pressure and is relevant to the human body. We recommend the BMI with a mean of 15.9 for children less than $\sim 8$ years and/or shorter than $\sim 1.3 \mathrm{~m}$; this category includes babies.

It appears urgent to study the intrinsic causes of overweight and obesity with the most accurate tools. The challenge is of great consequence to both the affected individual and society as a whole with burdens of large costs, both in money and Public Health efforts.

\section{Acknowledgments}

The authors would like to thank Dr. Stefan Engeli, Dr. Ralf Schleichert, Dr. Rüdiger Koch, Dr. Markus Büscher, Dr. Detlef Gotta, Mrs. Gisela Roes, and Mr. Peter Wieder for their kind help.

\section{References}

[1] J. M. Olefsky, Obesity. Harrison's Principles of Internal Medicine, McGraw-Hill, New York, NY, USA, 13th edition, 1994.

[2] R. J. Kuczmarski and K. M. Flegal, "Criteria for definition of overweight in transition: background and recommendations for the United States," American Journal of Clinical Nutrition, vol. 72, no. 5, pp. 1074-1081, 2000.

[3] W. C. Willett, W. H. Dietz, and G. A. Colditz, "Guidelines for healthy weight," The New England Journal of Medicine, vol. 341, no. 6, pp. 427-434, 1999.

[4] J. S. Flier, Adipositas. Harrison's Principles of Internal Medicine, McGraw-Hill, New York, NY, USA, 15th edition, 2001.

[5] P. Prasch, "Wenn Fett zur Sucht wird," Spektrum der Wissenschaft, vol. 7, pp. 86-87, 2003 (German).

[6] B. Bachtler, "Gewogen und zu schwer befunden," Spektrum der Wissenschaft, vol. 5, pp. 46-49, 2003 (German).

[7] K. Gesundheit, Gewicht und Größe, Kiepenheuer \& Witsch, Köln, Germany, 1992.

[8] D. A. Revicki and R. G. Israel, "Relationship between body mass indices and measures of body adiposity," American Journal of Public Health, vol. 76, no. 8, pp. 992-994, 1986.

[9] H. Stöcker, Taschenbuch der Physik, Harri Deutsch, Frankfurt, Germany, 1994.

[10] O. W. B. Schult, L. E. Feinendegen, W. W. Shreeve, and R. N. Pierson Jr., "Optimal use of weight and height for evaluation of obesity and other disorders," The International Journal of Body Composition Research, vol. 5, pp. 153-155, 2007.

[11] M. P. Pai and F. P. Paloucek, "The origin of the "ideal" body weight equations," Annals of Pharmacotherapy, vol. 34, no. 9, pp. 1066-1069, 2000.

[12] P. R. Bevington, Data Reduction and Error Analysis for the Natural Sciences, McGraw-Hill, New York, NY, USA, 1969.

[13] K. M. Flegal, "Ratio of actual to predicted weight as an alternative to a power-type weight-height index (Benn index)," American Journal of Clinical Nutrition, vol. 51, no. 4, pp. 540$547,1990$.

[14] W. W. Shreeve and R. N. Pierson Jr., "Element metabolism and body composition," in Molecular Nuclear Medicine, The Challenge of Genomics and Proteomics to Clinical Practice, L. E. Feinendegen, W. W. Shreeve, W. C. Eckelmann, Y. W. Bahk, and H. N. Wagner, Eds., pp. 252-300, Springer, Berlin, Germany, 2003.

[15] J. L. Baker, L. W. Olsen, and T. I. A. Sørensen, "Childhood body-mass index and the risk of coronary heart disease in adulthood," The New England Journal of Medicine, vol. 357, no. 23, pp. 2329-2337, 2007.

[16] C. D. Florey, "The use and interpretation of ponderal index and other weight-height ratios in epidemiological studies," Journal of Chronic Diseases, vol. 23, no. 2, pp. 93-103, 1970.

[17] T. Khosla and C. R. Lowe, "Indices of obesity derived from body weight and height," British Journal of Preventive \& Social Medicine, vol. 21, no. 3, pp. 122-128, 1967.

[18] F. J. Walther and L. H. J. Ramaekers, "The ponderal index as a measure of the nutritional status at birth and its relation 
to some aspects of neonatal morbidity," Journal of Perinatal Medicine, vol. 10, no. 1, pp. 42-47, 1982.

[19] J. G. Eriksson, T. Forsén, J. Tuomilehto, C. Osmond, and D. J. P. Barker, "Early growth and coronary heart disease in later life: longitudinal study," British Medical Journal, vol. 322, no. 7292, pp. 949-953, 2001.

[20] J. Fayyaz, "Ponderal index," Journal of the Pakistan Medical Association, vol. 55, no. 6, pp. 228-229, 2005.

[21] S. K. Bhargava, H. S. Sachdev, C. H. D. Fall et al., "Relation of serial changes in childhood body-mass index to impaired glucose tolerance in young adulthood," The New England Journal of Medicine, vol. 350, no. 9, pp. 865-875, 2004.

[22] B. R. Celli, C. G. Cote, J. M. Marin et al., "The body-mass index, airflow obstruction, dyspnea, and exercise capacity index in chronic obstructive pulmonary disease," The New England Journal of Medicine, vol. 350, no. 10, pp. 1005-1012, 2004. 


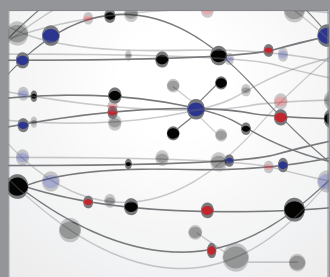

The Scientific World Journal
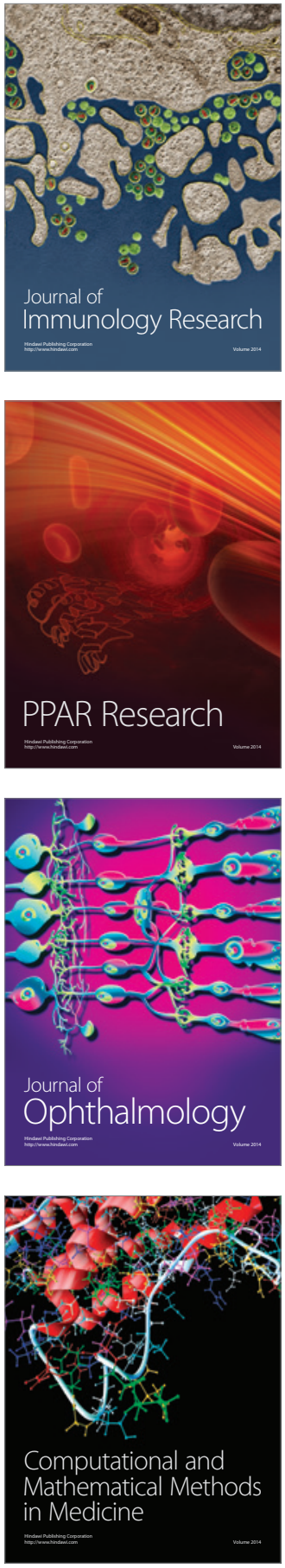

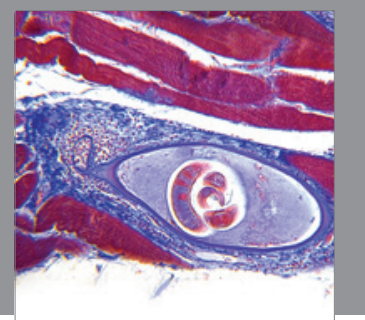

Gastroenterology

Research and Practice
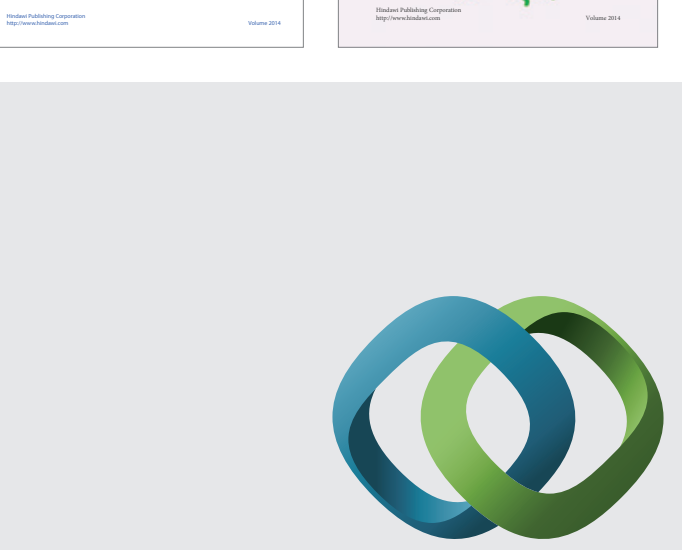

\section{Hindawi}

Submit your manuscripts at

http://www.hindawi.com
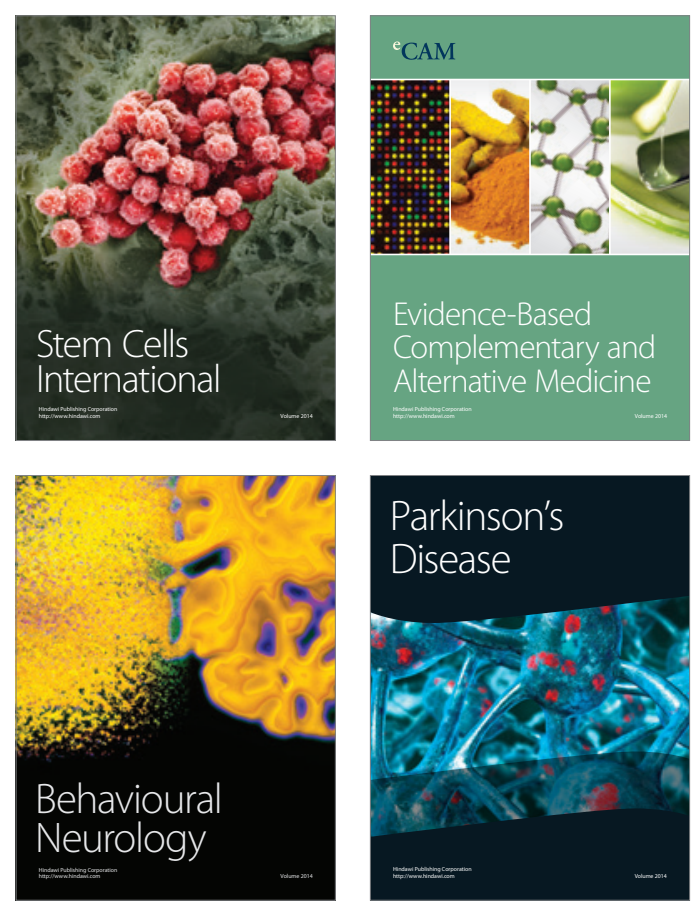

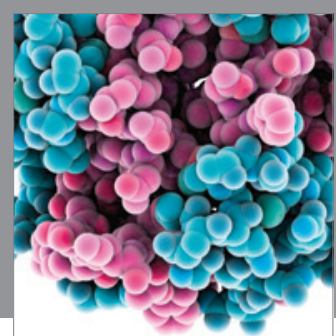

Journal of
Diabetes Research

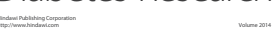

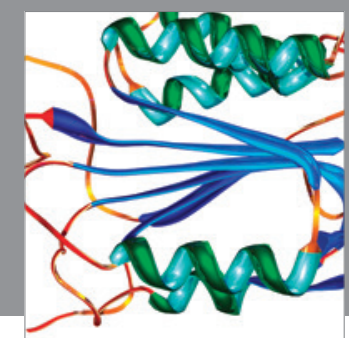

Disease Markers
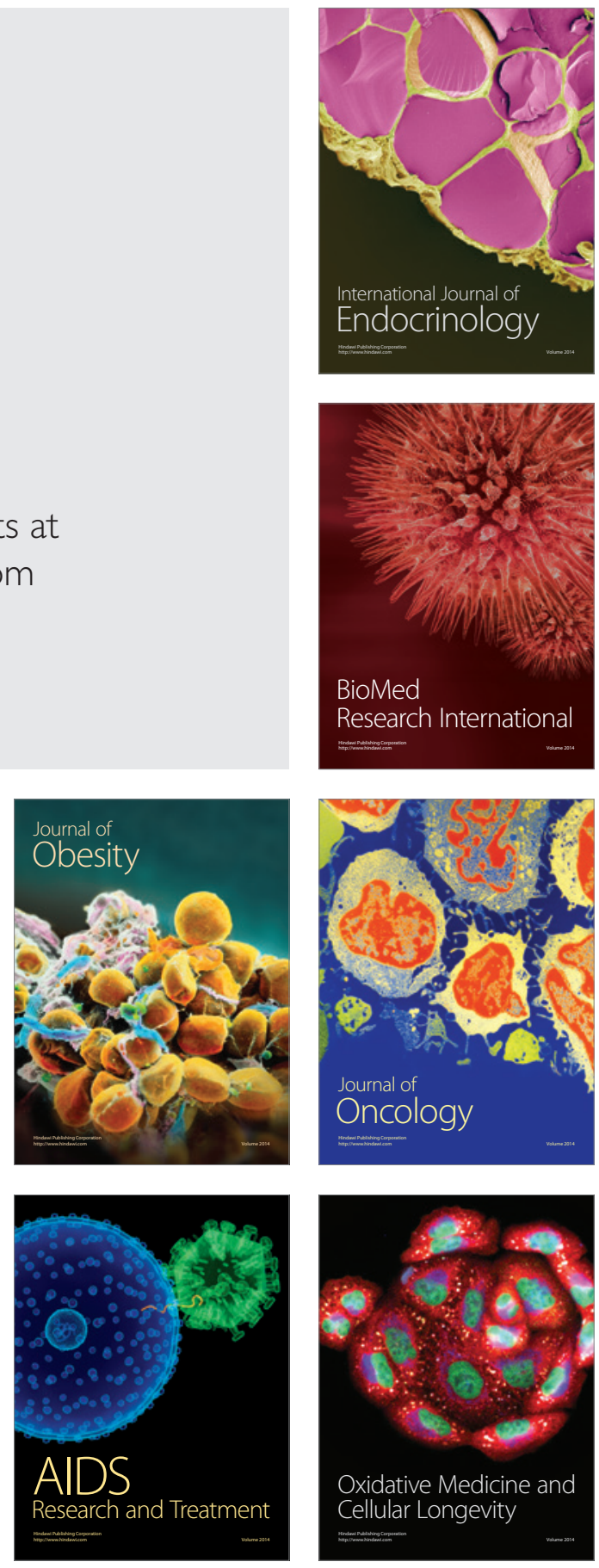